\title{
On the Exotic Hard X-ray Source Populations in the Hellas2XMM survey
}

\author{
C. Vignali \\ Dipartimento di Astronomia, Via Ranzani 1, 40127 Bologna, Italy \\ M. Mignoli \\ INAF-Osservatorio Astronomico di Bologna, Via Ranzani 1, 40127 Bologna, Italy \\ on behalf of the Hellas2XMM Collaboration ${ }^{1}$
}

\begin{abstract}
Recent hard X-ray surveys have proven to be effective in discovering large numbers of X-ray sources that, despite the likely association with active nuclei, appear to be characterized by "peculiar" properties. Among these "exotic" source populations, we will focus on the nature of two classes of hard X-ray sources: those characterized by high X-ray-to-optical flux ratios - a fraction of these are associated with the rather elusive Type 2 quasars - and the X-ray bright optically normal galaxies, also known as XBONGs.
\end{abstract}

Key words: Active Galaxies, X-rays, Surveys

\section{Introduction}

One of the most interesting results of recent X-ray surveys with Chandra and XMM-Newton consists in the discovery of a large number of sources with "peculiar" properties, i.e., not straightforwardly associated with Active Galactic Nuclei (AGN). In particular, two classes of sources will be discussed in the

Email addresses: cristian.vignali@unibo.it (C. Vignali), marco.mignoli@bo.astro.it (M. Mignoli).

1 A. Baldi, M. Brusa, N. Carangelo, P. Ciliegi, F. Civano, F. Cocchia, A. Comastri, F. Fiore, F. La Franca, R. Maiolino, G. Matt, S. Molendi, G.C. Perola, L. Pozzetti, S. Puccetti. 


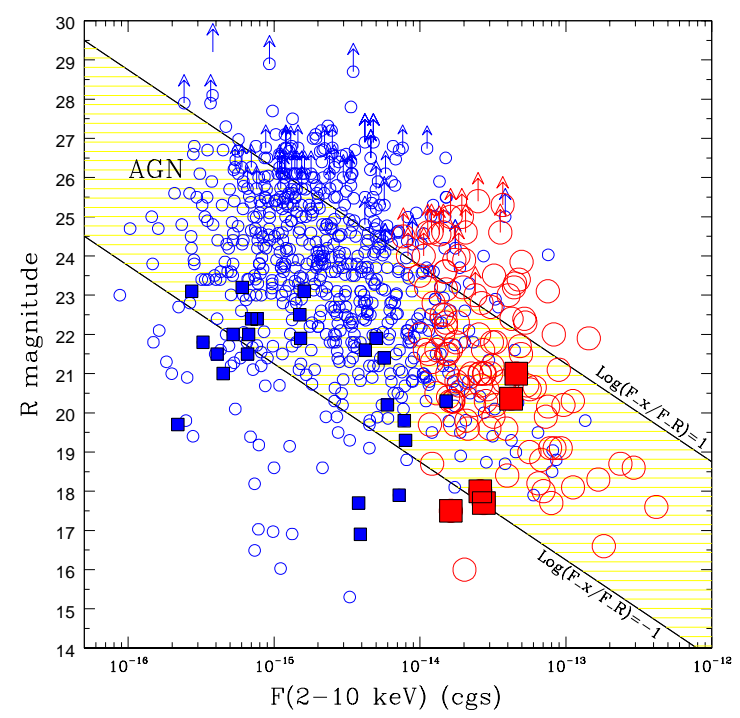

Fig. 1. 2-10 keV flux vs. $R$-band magnitude for sources detected by recent X-ray surveys [large symbols at bright X-ray fluxes: Hellas2XMM results; small symbols at faint X-ray fluxes: Chandra Deep Field-North and South (1; 2), Lockman Hole (3), ELAIS (4)]. The squares indicate the sources classified as XBONGs. "Normal" AGN typically populate the shaded region (within $\log F_{\mathrm{X}} / F_{\mathrm{R}}=\log (X / O) \pm 1$ ).

following: (a) sources with high X-ray-to-optical flux ratios (X/O) and (b) $\mathrm{X}$-ray bright optically normal galaxies (XBONGs).

\subsection{High X-ray-to-optical flux ratio sources}

In the $2-10 \mathrm{keV}$ flux vs. $R$-band magnitude plot (Fig. 1), the high $\mathrm{X} / \mathrm{O}$ sources populate the region above the dashed area (where AGN typically lie), being characterized by $\mathrm{X} / \mathrm{O} \geq 10$. The fraction of this source population among X-ray selected samples is $\approx 20 \%$ and constant over a broad range of X-ray fluxes [e.g., (5; 6) ] . From Fig. 1 it appears evident that a significant fraction of these high $\mathrm{X} / \mathrm{O}$ sources (typically those at $R>25$ ) cannot be spectroscopically identified even with 10-m class telescopes. We took advantage of the relatively bright X-ray flux limit of the Hellas2XMM survey $\left[F_{2-10 \mathrm{keV}} \approx 10^{-14} \mathrm{erg} \mathrm{cm}^{-2} \mathrm{~s}^{-1}\right.$; see (7) for details $]$ and, therefore, of the bright magnitudes of most of the optical counterparts, to shed light on the nature of these sources. We proceed through a two-steps method: firstly, we used FORS1 at the Very Large Telescope (VLT) to identify through spectroscopy the most luminous $(R<24)$ members of this population. Of the whole sample of 28 high X/O sources in the Hellas2XMM 1dF, 13 were targeted (5). Eight sources, at $z \approx 1-2$, match the Type 2 quasar optical definition (i.e., luminous sources with high-ionization, narrow emission lines). Four sources are broad-line (Type 1) AGN, while the remaining object is a moderate-luminosity 

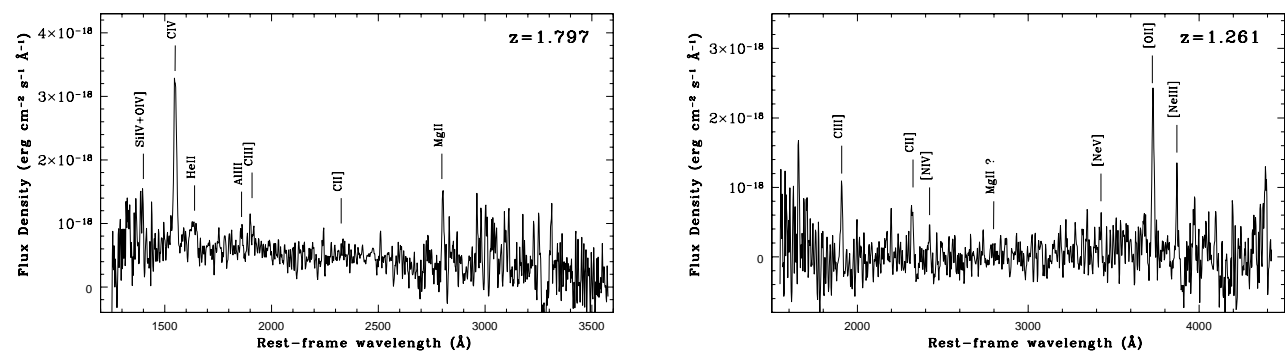

Fig. 2. Optical spectra of two Type 2 quasars discovered by the Hellas2XMM survey: source \#43 in PKS 0537-286 field (left panel) and source \#13 in Mrk 509 field (right panel). The principal emission lines are indicated.

emission-line galaxy. From the X-ray perspective, these objects match also the most widely accepted X-ray definition of Type 2 quasars, i.e., they are luminous $\left(L_{2-10 \mathrm{keV}} \gtrsim 10^{44} \mathrm{erg} \mathrm{s}^{-1}\right)$ and absorbed $\left[N_{\mathrm{H}} \gtrsim 10^{22} \mathrm{~cm}^{-2}\right.$; (8) $)$. In Fig. 2 two examples of Type 2 quasars from the Hellas2XMM surveys are shown. All the emission lines have $\mathrm{FWHM}<1,500 \mathrm{~km} \mathrm{~s}^{-1}$. Further information on the broad-band properties of these objects will be obtained with the planned sub-mm and near-infrared observations. Overall, although recent X-ray [e.g., $(2 ; 9 ; 10)]$ and optical surveys $[(11)$; see also $(12)$ and references therein] have proven to be effective in the detection of many Type 2 quasars, much work needs to be done at the faintest optical magnitudes. The second step of our approach addresses this point. Eleven faint $(R \gtrsim 24.5)$ high X/O sources were observed with ISAAC at the VLT in the $K_{\mathrm{S}}$ band. Bright $\left(K_{\mathrm{S}}<19\right)$ counterparts for 10 sources were found. All the counterparts have extremely red colors $(R-K>5.0)$; in particular, 7 sources are associated with massive bulge-dominated galaxies and have estimated redshifts in the range 0.9-1.4 [see (13) for details]. Since these objects are highly luminous and absorbed in the X-ray band, they show how effective the hard $X$-rays are in tracing obscured quasar accretion in massive ellipticals at $z \approx 1$-2. Two of the remaining counterparts are point-like, possibly AGN at $z \approx 1.6-2.4$, and only one object has a disk morphology (at $z \approx 0.8$ ).

\subsection{What's the nature of XBONGs?}

Although the XBONGs do not represent a new source population [see, e.g., (14)], only in the last few years it has become possible to unveil a significant number of these sources which were marginally represented by a few cases in past studies. Since the identification of the first XBONG detected in hard X-rays [the source P3 in (15) and (16); see Fig. 3 and 4], many more have been discovered (at $z \approx 0-1$ ) and studied. These sources are charac- 

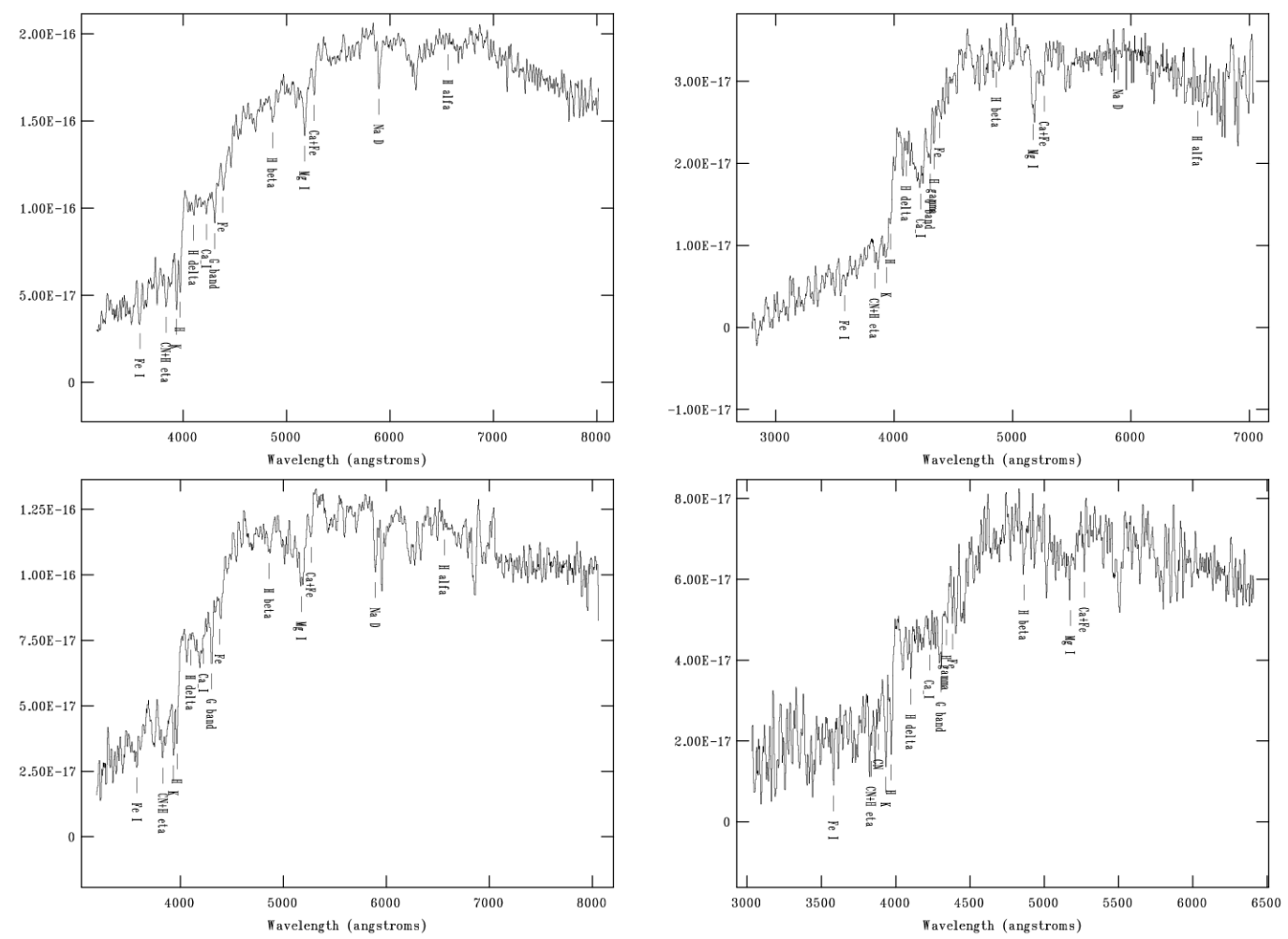

Fig. 3. Optical spectra of four XBONGs: (Top) source \#18 ( $z=0.159$, also known as "P3", left panel) and \#17 ( $z=0.320$, right panel), both in PKS 0312-770 field. (Bottom) source \#13 $(z=0.154)$ in Abell 2690 (left panel) and \#15 $(z=0.250)$ in Abell 1835 (right panel). The principal absorption lines are indicated.

terized by the lack of evident AGN signatures in moderate-quality optical spectra (see Fig. 3 for four examples from the Hellas2XMM survey), although X-rays provide indications for powerful $\left(L_{2-10 \mathrm{keV}}>10^{42} \mathrm{erg} \mathrm{s}^{-1}\right)$, not necessarily obscured, AGN emission. Several interpretations have been proposed to explain the observed properties of XBONGs, in particular: (a) they are the higher redshift counterparts of local Compton-thick galaxies (17). This has been suggested by the close resemblance of the spectral energy distribution of some XBONGs to that of the local Compton-thick galaxy NGC 6240; (b) the lack of spectral features at optical wavelengths is due to a combination of wide slits, limited-bandpass, low-resolution, and low signal-to-noise ratio spectra having the effect of diluting the nuclear emission (especially in the case of a low-luminosity AGN) by the host galaxy starlight $[(18 ; 19)$; see also $(20)$ ]; (c) the XBONGs are powered by advection-dominated accretion flows (ADAF) which are not effective in the production of the emission lines (21); (d) XBONGs can possibly be associated with BL Lac objects; this seems to be a viable explanation in at least one case (22). Whatever the explanation for the lack of spectral signatures, it appears difficult to ascribe their absence purely to the quality and bandpass of the observations. Although the dilution of the nuclear emission by the host galaxy could play an important role, it is a fact that the upper limits on typical AGN emission lines (i.e., [OIII] and 


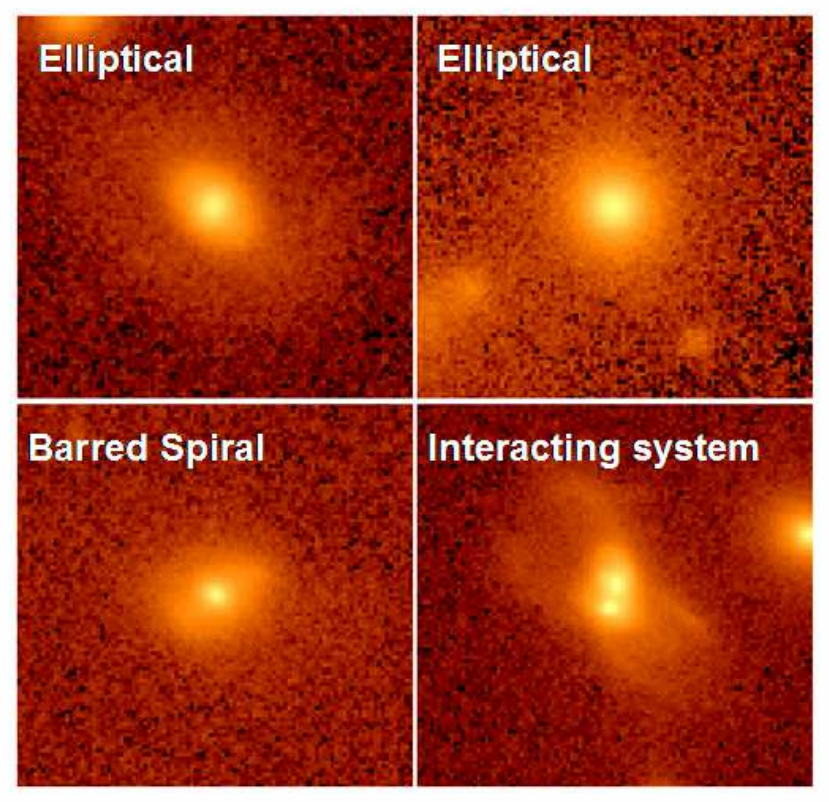

Fig. 4. $K_{\mathrm{S}}$-band images of the four XBONGs shown in Fig. 3 obtained with ISAAC at the VLT (seeing $\approx 0.45^{\prime \prime}$ ). The two galaxies of the interacting system are separated by $0.9^{\prime \prime}$, corresponding to $\approx 3.5 \mathrm{kpc}$.

$\mathrm{H} \alpha$ ) measured in the fairly good signal-to-noise ratio spectra of the XBONGs sampled by Hellas2XMM (see Fig. 3) are an order of magnitude less than the fluxes expected from the observed X-ray luminosity.

Finally, further evidence of the large variety in the properties of XBONGs comes from near-infrared (NIR) imaging [see Fig. 4 and (23)]. Despite the optical spectra, which are all typical of early-type galaxies, the NIR images show different morphologies: two are ellipticals, one is a barred spiral, and one consists of two close interacting galaxies (see Fig. 4.) Further broad-band studies and, possibly, a more effective definition of "xbongness" (e.g., a limit to the equivalent width of any optical emission line) are required to understand this "exotic" source population in more detail.

\section{References}

[1] Barger, A.J., et al. Optical and infrared properties of the 2 Ms Chandra Deep Field North X-ray sources. Astron. Jou. 126, 632, 2003.

[2] Szokoly, G.P., et al. The Chandra Deep Field South: optical spectroscopy I. Astroph. Jou. Suppl. Ser. 155, 271, 2004.

[3] Mainieri, V., et al. XMM-Newton observation of the Lockman Hole. II. Spectral analysis. Astron \& Astroph. 393, 425, 2002. 
[4] Manners, J.C., et al. The ELAIS deep X-ray survey - I. Chandra source catalogue and first results. Mon. Not. Roy. Astron. Soc. 343, 293, 2003.

[5] Fiore, F., et al. The Hellas2XMM survey. IV. Optical identifications and the evolution of the accretion luminosity in the Universe. Astron \& Astroph. 409, 79, 2003.

[6] Eckart, M.E., Laird, E.S., Stern, D., Mao, P.H., Helfand, D.J., Harrison, F.A. The serendipitous extragalactic X-ray source identification (SEXSI) program. II. Optical imaging. Astroph. Jou. Suppl. Ser. 156, 35, 2004.

[7] Baldi, A., Molendi, S., Comastri, A., Fiore, F., Matt, G., Vignali, C. The Hellas2XMM survey. I. The X-ray data and the log N-Log S relation. Astroph. Jou. 564, 190, 2002.

[8] Perola, G.C., et al. The Hellas2XMM survey. VI. X-ray absorption in the $1 \mathrm{dF}$ AGN sample through a spectral analysis. Astron \& Astroph. 421, 491, 2004.

[9] Norman, C., et al. A classic Type 2 QSO. Astroph. Jou. 571, 218, 2002.

[10] Stern, D., et al. Chandra detection of a Type II quasar at $z=3.288$. Astroph. Jou. 568, 71, 2002.

[11] Zakamska, N.L., et al. Candidate Type II quasars from the Sloan Digital Sky Survey: I. Selection and optical properties of a sample at $0.3<z<0.83$. Astron. Jou. 126, 2125, 2003.

[12] Vignali, C., Alexander, D.M., Comastri, A. Evidence for X-ray obscuration in Type II quasar candidates from the Sloan Digital Sky Survey. Mon. Not. Roy. Astron. Soc. 354, 720, 2004.

[13] Mignoli, M., et al. The Hellas2XMM survey. V. NIR observations of X-ray sources with extreme X/O ratios. Astron \& Astroph. 418, 827, 2004.

[14] Elvis, M., Schreier, E., Tonry, J., Davis, M., Huchra, J. Two optically dull galaxies with strong nuclear X-ray sources. Astroph. Jou. 246, 20, 1981.

[15] Fiore, F., et al. Spectroscopic identification of ten faint hard X-ray sources discovered by Chandra. New Astronomy 5, 143, 2000.

[16] Comastri, A., et al. The Hellas2XMM survey. II. Multiwavelength observations of P3: an X-ray bright, optically inactive galaxy. Astroph. Jou. 571, 771, 2002.

[17] Comastri, A., Brusa, M., Mignoli, M. Unconventional AGN in hard X-ray surveys. Astron. Nach. 324, 28, 2003.

[18] Moran, E.C., Filippenko, A.V., Chornock, R. "Hidden" Seyfert 2 galaxies and the X-ray background. Astroph. Jou. 579, L71, 2002.

[19] Severgnini, P., et al. XMM-Newton observations reveal AGN in apparently normal galaxies. Astron \& Astroph. 406, 483, 2003.

[20] Moran, E.C., et al., these proceedings.

[21] Yuan, F., Narayan, R. The Nature of X-ray bright optically normal galaxies. Astroph. Jou. 612, 724, 2004.

[22] Brusa, M., et al. The Hellas2XMM survey. III. Multiwavelength observations of hard X-ray selected sources in the PKS 0312-77 field. Astron \& Astroph. 409, 65, 2003.

[23] Mignoli, M., et al., in preparation. 